\title{
Perfil del turista nacional que consume alimentos durante Semana Santa en Mazatlán, Sinaloa
}

\author{
Silvestre Flores Gamboa* \\ Universidad de Occidente \\ Campus Mazatlán \\ Christian Andrew Solorzano \\ Universidad Autónoma de Durango, Campus Mazatlán
}

\section{Resumen}

El presente artículo pretende establecer los patrones de consumo de alimentos del turista nacional que vacaciona en Mazatlán, Sinaloa, durante la Semana Santa, lo cual incluye las predilecciones gastronómicas, los restaurantes y el gasto que esto representa. El analisis se realiza con base en un conjunto de variables cuantitativas, obtenidas a través de la encuesta como instrumento de medición. A la luz de los resultados se describe la importancia de la gastronomía como parte de la oferta que brinda el destino a sus visitantes, a través del disfrute de las tradiciones culinarias del puerto, entre las que sobresalen los alimentos elaborados a base de pescados y mariscos.

\section{Palabras clave}

Mazatlán, Semana Santa, gastronomía, perfil de turista, turista nacional.

Recibido: 12/09/2012 · Aceptado:05/10/2012 


\title{
National Tourist Profile of food consumption during Easter Week in Mazatlan, Sinaloa
}

\author{
Silvestre Flores Gamboa \\ Universidad de Occidente \\ Campus Mazatlán \\ Christian Andrew Solorzano \\ Universidad Autónoma de Durango, Campus Mazatlán
}

\begin{abstract}
This paper intends to establish the dietary intake patterns of national tourists who spend their holidays in Mazatlan, Sinaloa, during Easter week; which includes gastronomic preferences, choice of restaurants and the expenditure allocated to it. The analysis is based on a set of quantifiable variables drawn from a series of surveys considered as a measurement instrument. It highlights the importance of gastronomy as an offer for visitors, through the pleasure of the culinary tradition in the port, in which fish and seafood stand out.
\end{abstract}

\section{KeY WORDS}

Mazatlan, Easter, gastronomy, tourist profile, national tourism.

Recibido: 21/08/2012 · Aceptado: 21/11/2012 


\section{Introducción}

La actividad turística forma parte de los principales sectores económicos de México, no solo por el efecto financiero que produce, sino también por la interacción social y cultural que en ella se alcanza. Existen múltiples y variados efectos para la población que habita en un destino turístico, por ejemplo, un aumento de la calidad de vida de los habitantes, la creación de empleos, la conservación de las tradiciones culturales, de los atractivos naturales y del medio ambiente, la difusión y el consumo de alimentos que representan parte de la gastronomía regional, entre otros.

Los turistas que visitan un destino turístico determinado pueden tomar innumerables decisiones durante su viaje: cancelar la visita a un atractivo, asignar cierta cantidad monetaria a la compra de recuerdos del sitio, pagar un tour o cambiar el medio de transporte en sus traslados, etc. Sin embargo, durante su estancia no pueden dejar de alimentarse.

Mazatlán, como todo destino turístico, necesita una serie de componentes que lo hagan funcional y exitoso, en otras palabras, debe contar con una planta turística adecuada, entendida esta como "la conformación de todas aquellas instalaciones y el equipo que produce los bienes y servicios que necesita el turista durante su estancia y desplazamiento" (Gurría Di-Bella, 1994: 52). Por lo tanto, debe contar con las suficientes instalaciones, equipos y servicios que le permitan ofrecer a los visitantes todo lo indispensable para que su estadía sea placentera. En este orden de ideas queda claro que los servicios de alimentación (restaurantes, fondas, etc.) son fundamentales.

Ahora bien, durante la última década la afluencia de visitantes a Mazatlán ha sido estudiada básicamente a través de tres grandes perspectivas, la histórica, la económica y la social. La primera abarca desde el nacimiento de la ciudad como destino turístico hasta su desarrollo contemporáneo (Santamaría Gómez, 2002 y 2005); en cuanto a lo económico, destacan aquellos estudios enfocados en las inversiones realizadas dentro del sector (Santamaría Gómez y Espinoza García, 2011) y el ciclo de vida del destino (Santamaría Gómez y Barbosa Jasso, 2006), mientras que en el aspecto social sobresalen los análisis respecto al papel que juega la migración (Lizárraga Morales, 2005 y 2007) y la violencia (Santamaría Gómez y Flores Gamboa, 2012). 
Los estudios realizados durante la Semana Santa en este destino son escasos, uno de ellos analiza desde un punto de vista etnográfico una parte de las vivencias observadas en los visitantes a través de sus pautas de comportamiento (Santoyo Parroquín, 1999: 217), mientras que otro aborda la experiencia del turismo cultural en visitantes nacionales y extranjeros tomando como punto de referencia de este segmento el centro histórico de la ciudad (Gamboa Mora, 2007: 40); sin embargo, la investigación relacionada con las actividades culinarias es prácticamente nula.

El presente estudio destaca la importancia de conocer las pautas, preferencias, establecimientos y el gasto en alimentación del turista en un destino tradicional de sol y playa como Mazatlán, Sinaloa, durante una temporada vacacional en particular: Semana Santa. Por esta razón resulta interesante conocer icuál es la actitud que toma un turista hacia la gastronomía local y regional?, ¿cuáles son sus preferencias gastronómicas?, así como ¿qué tipo de establecimientos de consumo de alimentos utiliza para satisfacer esta necesidad?, y ¿qué porcentaje de su gasto lo destina al consumo de alimentos?

\section{Antecedentes}

En el contexto actual del negocio de los viajes y el turismo, todo consumidor de productos turísticos posee una gran variedad y una creciente capacidad de elección al momento de planear o realizar su viaje. En este mismo sentido, también son diversos los factores que motivan a una persona a decidirse por determinado viaje, destino, hotel, atractivo o servicio, incluso "viajar puede ser una de las diversas maneras de satisfacer una necesidad” (Holloway, 1997: 87).

Las necesidades sugieren que los productos que solicita cada individuo son indispensables para su vida diaria, aunque evidentemente no sea el caso de los productos turísticos, en los cuales solo se expresa el deseo de un mayor número de bienes y servicios; ello como una característica de la sociedad orientada hacia el consumo.

Por otro lado, la motivación y el propósito están estrechamente ligados (Holloway, 1997: 85), por lo que es primordial la percepción, entendida como aquel "conjunto de mecanismos y procesos a través de los cuales el organismo adquiere conocimiento del mundo y de su entorno, basándose en información elaborada por sus sentidos, la misma puede darse por tratamientos guiados por 
los estímulos y otros guiados por las representaciones o conceptos ya existentes" (Jones et al., cit. en Rodríguez, Requena y Muñoz, 2009: 135).

La percepción de cualquier individuo sobre unas vacaciones o cualquier otro producto es afectada por sus experiencias y actitudes, por lo que "si la percepción de la necesidad y de la atracción se iguala, el consumidor estará motivado a comprar el producto" (Holloway, 1997: 87), en otras palabras, a visitar determinado destino o consumir un producto o servicio turístico durante su estancia.

Como se comentó en párrafos anteriores, una persona puede estar motivada por una gama de productos y/o servicios turísticos que le ayuden a cubrir sus principales necesidades de descanso, recreo, esparcimiento, negocios, etc. Por ello, las empresas que se dedican a esta actividad tienen un objetivo primordial: satisfacer al turista, pero para lograrlo de forma exitosa deben conocer lo mejor posible sus necesidades. Esto da pie a que los viajeros se sitúen en grupos de comportamiento homogéneo (tipos de turismo), con necesidades muy concretas, y programar sus viajes adaptados a esos requerimientos.

Cabe mencionar que el turismo puede ser clasificado de diversas formas, una de las más comunes es hacerlo desde el punto de vista de la "motivación" que pueden tener las personas en su decisión de realizar un viaje turístico. Renato Quesada Castro (2007: 101-107) describe adecuadamente una de estas clasificaciones y agrupa los siguientes tipos de turismo:

- Turismo cultural. Busca el disfrute y permanencia en sitios donde el arte y la cultura se manifiestan notoriamente por encima de otros recursos turísticos.

- Turismo deportivo. Es motivado por tres circunstancias distintas: la participación como espectadores, para aprender, disfrutar o practicar una actividad deportiva o bien en la participación de una competencia.

- Turismo de placer. Su principal motivación es divertirse y disfrutar a plenitud el sitio visitado. De hecho, este segmento es un turismo masivo pues la mayoría de las personas viaja con el propósito de hacer en el destino lo que les gusta (broncearse, jugar, comprar, etcétera).

- Turismo de salud. Los individuos viajan a un lugar caracterizado por sus condiciones y facilidades para el descanso y la recuperación, o para recibir un tratamiento o intervención quirúrgica. 
- Turismo de congresos y convenciones. Son aquellos viajes para asistir a reuniones de diversa índole, donde se combinan las sesiones de trabajo con los recorridos turísticos que se programen, o bien con las actividades o tours que se ofrecen como opcionales.

- Turismo de negocios. Son aquellos viajes a otro lugar diferente del que se tiene la residencia por gestiones o labores propias de una empresa.

- Turismo naturalista. Los individuos son atraídos o motivados por el afán de conocer, disfrutar o estudiar un recurso natural singular o característico de un destino. Cabe mencionar que, según sea el caso de interés y de identificación será su nivel de especialización, por lo que puede subdividirse a su vez en otros.

Diversos autores consideran la existencia de otros tipos de turismo en sus respectivas clasificaciones, sin embargo, y tomando en cuenta el objetivo del presente estudio, es preciso indicar que dentro del turismo cultural se manejan diversos subsegmentos, entre los que destacan el turismo familiar (visita a familiares y amigos), el turismo religioso (peregrinaciones o visita de lugares religiosos o templos), el turismo étnico (en sitios donde reside un grupo étnico en particular) y el turismo gastronómico (viajes a lugares destacados por su cocina) (Quesada Castro, 2007: 102), al que nos referiremos con mayor profundidad más adelante.

\section{Importancia de la gastronomía en el turismo}

La alimentación puede ser analizada desde varias perspectivas, por un lado, "cumple con una función biológica al suministrar al cuerpo las sustancias indispensables para su subsistencia” (Schlüter, 2006: 43), también es visualizada como un "proceso voluntario, consciente y educable" (Martínez Jasso y Villezca Becerra, 2005: 197). Desde el contexto cultural, "la alimentación es un factor de diferenciación cultural que permite a todos los integrantes de una cultura, sin importar su nivel de ingresos, manifestar su identidad” (González Turmo, 1999: 246).

Ahora bien, desde el contexto del turismo, alimentarse también forma parte de la experiencia turística de todos los visitantes al hacer uso de los servicios de alimentación de una localidad específica (Nunes dos Santos, 2007: 239), además representa una forma de vinculación entre las personas y los territorios que visitan (Clemente Ricolfe et al., 2008: 190). 
Asimismo, la alimentación en cuanto fuente de placer posibilita que la gastronomía se constituya como un segmento y atractivo turístico (Nunes dos Santos, 2007: 239; Clemente Ricolfe et al., 2008: 190), razón por la cual esta cobra cada vez mayor relevancia a nivel mundial. Efectivamente, "los turistas podrían viajar sin visitar un monumento o lugar, pero nunca sin comer. Posiblemente, se recuerde un lugar primero por lo que se comió, y luego por lo que se vio. E incluso, cada día crece el número de personas que acuden a un destino concreto sólo para conocer y degustar su oferta gastronómica” (Clemente Ricolfe et al., 2008: 189).

Es preciso mencionar que no se puede concebir de la misma forma a la persona que viaja y come, la cual "no manifiesta un interés especial por la oferta gastronómica local pero deseando que la misma se adapte a sus necesidades y preferencias en el destino vacacional", que al turista que viaja exclusivamente para comer (Torres Bernier, cit. en Schlüter, 2006: 112). Por lo tanto, el turismo gastronómico es concebido como "una actividad del turista o visitante que planea sus viajes parcial o totalmente para degustar la gastronomía del lugar o realizar actividades relacionadas con la gastronomía” (Flavián Blanco y Fandos Herrera, 2011: 14).

De esta forma, el turismo gastronómico se ha consolidado como un nuevo tipo de turismo, cuya finalidad es el conocimiento y disfrute de la gastronomía de un sitio en particular (Clemente Ricolfe et al., 2008: 190). Además, este segmento turístico "puede ayudar a rescatar antiguas tradiciones que se encuentran en el camino de la desaparición” (Schlüter, 2006: 42), ya que la gastronomía "forma parte de la cultura de los pueblos y no sólo nutre al cuerpo sino también al espíritu” (2006: 43).

En la actualidad, no solo se reconoce la importancia socioeconómica y cultural de la gastronomía para un pueblo, región o país, sino que también, desde el punto de vista del turismo gastronómico, esta posee y genera diferentes atributos positivos, entre los cuales destacan los siguientes:

- Fenómeno local de alcance universal

- Incidencia favorable sobre la economía, el empleo y el patrimonio local

- Capacidad de expansión como motivo principal de viaje turístico

- Contribuye a la mejora de la percepción general del destino (Blanco, 2010) 
De igual manera, esta situación ha favorecido diversas actividades vinculadas con la gastronomía de una localidad o región, como por ejemplo "rutas alimentarias, turismo del vino, visita a ferias gastronómicas y mercados, comida en restaurantes típicos de la ciudad, etc.” (Clemente Ricolfe et al., 2008: 190). Por lo tanto, dentro del negocio de los viajes y el turismo es posible utilizar estos "elementos gastronómicos como atractivos turísticos" (Nunes dos Santos, 2007: 239).

\section{Perfil del turista y gasto en el consumo de alimentos}

Ante la importancia creciente de la gastronomía como parte de la oferta turística, diversos destinos realizan programas para mejorar su cocina típica, lo que implica tanto la preparación de los platos como la calidad del servicio en los diferentes establecimientos de alimentos y bebidas, buscando, entre otras cosas, consolidar una imagen turística que sirva para incentivar a las personas a trasladarse a ese destino, así como motivar un aumento en la proporción del gasto orientado al consumo de alimentos.

En México, en los últimos cinco años se han incrementado los estudios relacionados con el perfil del turista en destinos de sol y playa a través de algunas pautas afines con su experiencia y tipo de consumo. Por ejemplo, en la isla caribeña de Cozumel, Quintana Roo, se trabajó el perfil del turista internacional a partir de su capital simbólico (Anaya Ortiz y Palafox Muñoz, 2010: 171). En Acapulco, Guerrero, se analizó el perfil de los turistas que visitan el puerto en términos de su satisfacción sobre las ofertas de mercado (Guerrero Ocampo, Santiago Moreno y Bouza Herrera, 2009: 77).

A nivel nacional, el Centro de Estudios Superiores en Turismo (Cestur), órgano desconcentrado de la Secretaría de Turismo (Sectur), es la institución que ha abarcado con mayor extensión y profundidad teórica los estudios sobre el perfil del turista, ya que no solo abordan diversas variables de estudio y segmentos de mercado específicos, sino que también se extienden a diversos destinos turísticos del país. De hecho, desde 2001 se ha llevado a cabo en diferentes momentos el estudio de Perfil y grado de satisfacción de los turistas, el cual también sirve como plataforma para otras investigaciones similares, como el Análisis de la lealtad del consumidor de destinos turísticos en México (Cestur, 2011), cuyo propósito es identificar los insumos necesarios para la generación de un modelo de lealtad en México. 
Sin embargo, en México son relativamente escasos los estudios publicados sobre el perfil del turista conforme a su tipo de consumo gastronómico y gasto turístico, entendido este como "la totalidad del dinero desembolsado por los turistas en la contratación de su viaje y durante toda su estancia en su lugar de destino" (Cabarcos Novás, 2006: 6). Uno de los ejercicios a destacar en este ámbito es la Encuesta del Gasto en Turismo en Hogares 2011, publicada por la Secretaría de Turismo (Sectur, 2011), que describe de forma general las principales áreas del gasto efectuado por un turista y excursionista nacional.

Por tal razón, el presente estudio contribuye al conocimiento generado hasta hoy en esta área, sobre todo cuando a nivel nacional se ha destacado la relevancia que tiene el consumo turístico en el sector económico mexicano, donde "ocho de cada diez turistas son consumidores nacionales, es decir, el consumo generado por el turismo doméstico es casi seis veces superior al efectuado por el turismo internacional” (Sectur, 2011: 2).

\section{Mazatlán, turismo y gastronomía}

El puerto de Mazatlán es considerado el mayor destino turístico en el estado de Sinaloa (Santamaría Gómez y Flores Gamboa, 2012: 6), en él se concentra la mayor parte de la infraestructura hotelera, con un total de 177 establecimientos de hospedaje de los 419 existentes en todo el territorio sinaloense, que en conjunto suman 10101 cuartos disponibles registrados hasta 2009 (INEGI, 2010). Asimismo, de acuerdo con el sistema Datatur de la Sectur, durante 2011 la actividad hotelera del destino registró un total de 1548300 turistas en sus centros de hospedaje, de los cuales 378624 son de origen extranjero, mientras que 1169676 fueron de procedencia nacional, conformando en la actualidad su mayor mercado.

No obstante que en los últimos años se han realizado diversas acciones enfocadas a resaltar la gastronomía regional sinaloense y la mazatleca entre los turistas que visitan el destino, esto solo se efectúa como parte de su oferta turística y no ha sido aprovechado plenamente para generar un nuevo segmento turístico, en este caso el turista gastronómico. Un ejemplo de esto se observó durante los festejos del Día Mundial del Turismo, celebrado en el puerto el día 27 de septiembre de 2011 en presencia de la secretaria de Turismo, Gloria Guevara Manzo; del presidente de la nación, Felipe Calderón Hinojosa; del gobernador 
constitucional de Sinaloa, Mario López Valdez; del alcalde de Mazatlán, Alejandro Higuera Osuna, entre otras personalidades, cuando Mazatlán fue facilitador y testigo de un récord Guinnes al elaborarse el "cóctel de camarón más grande del mundo”, con un peso total de 538.5 kilogramos, acción que buscó dar al puerto un realce mediático nacional e internacional.

El año 2012 ha sido uno de los más intensos en cuanto al turismo y la gastronomía, pues el 28 y 29 de febrero se realizó por vez primera en Mazatlán una de las etapas (semifinales) del certamen "Cocinero del Año México", cuyo objetivo es promover la gastronomía mexicana, prestigiar y promocionar la profesión de cocinero y apoyar el turismo gastronómico en México. De igual manera, se organizó la edición número 26 de la tradicional "Feria del Pescado", que se instala año con año durante Semana Santa con el fin de que los visitantes consuman los diferentes platillos elaborados a base de mariscos o pescado y disfruten su buen olor, sabor y precio.

A finales de octubre la Secretaría de Turismo del Estado de Sinaloa organizó por vez primera el "Foro Gastronómico: Así sabe Sinaloa", en el que destacó la participación de reconocidos cocineros de origen nacional e internacional, la instalación de pabellones alimentarios, tianguis gourmet, zonas para cierre de negocios, entre otras actividades. Todo ello para resaltar el valor de la gastronomía sinaloense y posicionar al estado como un productor alimentario de características únicas.

Este tipo de actividades desarrolladas en el puerto de Mazatlán durante 2012 demuestran el aumento en su interés por utilizar la gastronomía como un fuerte atractivo turístico entre los miles de visitantes de origen nacional e internacional que año con año llegan a su territorio.

\section{Metodología}

De acuerdo con la relevancia y el contexto del presente estudio se plantearon como objetivos conocer las preferencias en el consumo de determinados alimentos por parte del turista de origen nacional que visita Mazatlán durante Semana Santa, así como el tipo de establecimientos utilizados y el gasto turístico que le asigna a su consumo. Los métodos, técnicas e instrumentos para recolectar los datos y cumplir con los objetivos propuestos están cimentados en técnicas de investigación documental y de campo bajo un enfoque cuantitativo. 
En cuanto a la técnica de investigación de campo se diseñó una encuesta que consta de 17 preguntas agrupadas en cuatro secciones. La primera recopila información sociodemográfica de los encuestados (procedencia, sexo y edad con base en tres rangos de edades: 18-30, 31-45, 46 y más años).

En la segunda sección se hace referencia al motivo del viaje, el medio de transporte empleado para su traslado y a las personas con las que lo realiza. La tercera sección comprende el consumo de alimentos durante la estancia en el destino turístico, así como el nivel de los gastos que realizan en esta actividad.

La última sección hace énfasis en las expectativas de los turistas sobre Mazatlán en cuanto a la gastronomía y el consumo de alimentos. Cabe mencionar que dentro de este apartado, en la pregunta final se aplicó la escala de Likert de cinco puntos con la intención de medir la valoración dada por los encuestados para obtener un promedio que permitiera conocer el grado de satisfacción en las diferentes expectativas planteadas, como la calidad de los alimentos, el servicio otorgado por el personal, los precios, las porciones de comida, etcétera.

El muestreo es de tipo probabilístico, donde, según el marco muestral (turistas de origen nacional que visitan Mazatlán), todas las personas tienen la oportunidad de ser seleccionadas de forma aleatoria. Por otro lado, la técnica con que se obtuvo información fue la encuesta escrita para ser respondida por los seleccionados, la duración promedio de aplicación fue de 12 minutos, por lo que la forma en que se aplicó el instrumento fue por entrevista personal a un total de 100 visitantes.

El procesamiento de los datos recopilados mediante la encuesta se desarrolló en tres etapas: la tabulación de los datos en función de las preguntas realizadas, el cálculo de las respuestas (porcentajes), con ayuda del programa Excel 2007, y el análisis, interpretación y descripción de los resultados reflejados en cuadros y gráficas, respecto del marco teórico y los antecedentes de investigación.

El estudio solo considera a turistas de origen nacional de ambos sexos y mayores de 18 años que pasaron más de 24 horas en la ciudad de Mazatlán, esto último tomando en cuenta la definición de Manuel Gurría Di-Bella en su obra Introducción al turismo, donde señala que un turista es "un visitante temporal que permanece cuando menos 24 horas en el país visitado” (1994: 16). Asimismo, los instrumentos se aplicaron en los principales sitios frecuentados por turistas (playas, malecón, centro histórico y acuario), durante el periodo 
comprendido en los días de Semana Santa de 2012 (Jueves y Viernes santos, Sábado de Gloria y Domingo de Resurrección), variando los horarios y lugares de la encuesta.

\section{Resultados}

La primera parte del cuestionario aplicado a turistas de origen nacional durante la Semana Santa del 2012 en Mazatlán mostró que la mayoría de ellos procedía de algunos municipios sinaloenses (23\%), así como de los estados de Sonora (13\%), Baja California (13\%), Coahuila (9 \%), Jalisco (8 \%), Nuevo León (7 $\%)$ y Chihuahua (6 \%). Por otra parte, $59 \%$ de las personas pertenece al sexo masculino, mientras que el restante (41 \%) al sexo femenino. En cuanto al promedio de edad, $63 \%$ tenía entre 18 y 30 años, mientras que $30 \%$ oscilaba entre 35 y 45 años, y solo 7 \% tenía más de 45 años cumplidos.

En la segunda sección del cuestionario, enfocada a conocer los motivos, los medios y el acompañamiento de los visitantes a Mazatlán, 37 \% de las personas encuestadas reportó que visitaba el puerto por primera vez, mientras que la gran mayoría, es decir $63 \%$, ya lo conocía, lo que refleja el posicionamiento y la preferencia por este destino entre los entrevistados. Esta situación también puede ser explicada desde la perspectiva de la lealtad del consumidor de acuerdo con el estudio de Cestur (2011: 17), donde $91.3 \%$ de los 864 turistas entrevistados que ya conocía el destino opinó que en definitiva sí volvería.

En lo referente al medio de transporte utilizado para llegar a Mazatlán, destaca que $48 \%$ de los turistas nacionales arribó por carretera (48 \% automóvil y $28 \%$ autobús), y solo $20 \%$ reportó haber llegado vía aérea. Los restantes utilizaron una embarcación o motocicletas. En cuanto al motivo principal de su visita al puerto, la gran mayoría (92\%) fue vacacional, mientras que un reducido número lo hizo por motivos familiares o de negocios. A su vez, $57 \%$ de ellos lo hizo acompañado por familiares, $31 \%$ con amigos, $9 \%$ manifestó haber arribado solo y $3 \%$ con otro tipo de acompañamiento.

Dentro de la tercera sección del cuestionario, el resultado de las preguntas tiene relación directa con el papel de la gastronomía a partir de su consumo, por lo que al preguntar a los turistas dónde acostumbran consumir alimentos durante su estancia en Mazatlán y sus principales razones para hacerlo ahí, la gran mayoría prefirió un restaurante (68 \%) para consumir alimentos, mientras que 
los demás optaron por puestos ambulantes (12\%), dentro de los hoteles (8\%), centros comerciales (6\%) y mercados (2\%). El resto manifestó hacerlo en otros sitios como casas de familiares.

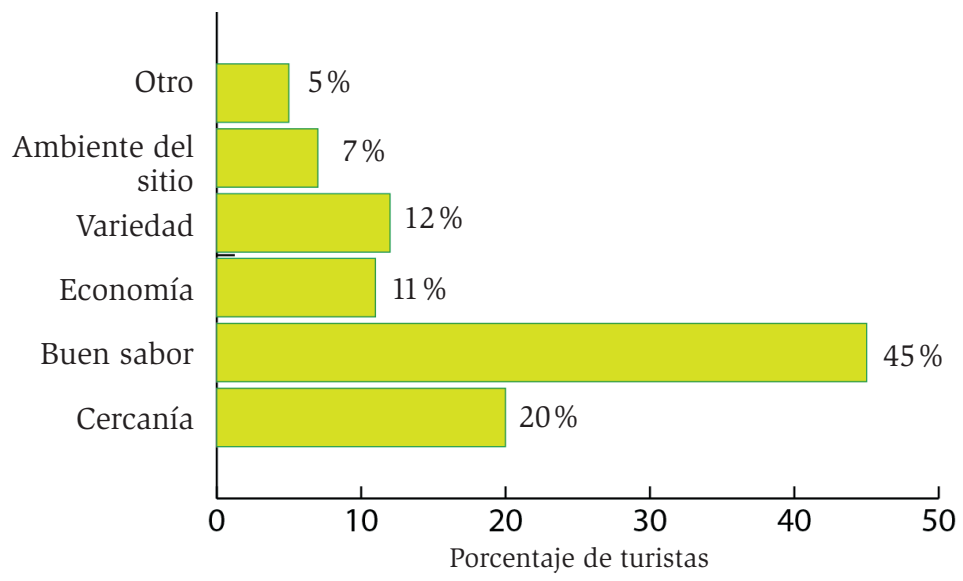

GrÁfICA 1. Razones para consumir alimentos en los sitios

La gráfica 1 muestra que entre los principales motivos para consumir alimentos en restaurantes se encuentra el buen sabor (45\%) y la cercanía (20 $\%$ ), mientras que en menor término su variedad gastronómica (12 \%), el factor económico (11 \%) y el ambiente del sitio (7 \%), otros (5\%). Este resultado revela la importancia del paladar en la decisión de los turistas, pues el aspecto geográfico del lugar, el gasto económico e inclusive la variedad y el ambiente del local son factores que inciden en menor medida al tomar su decisión.

Por su parte, en cuanto al tipo de alimentos que los turistas consumen generalmente durante su estancia en el destino sinaloense, destacan los elaborados a base de pescados y mariscos (65\%). El resto de los turistas nacionales entrevistados prefirió el consumo de carnes (21 \%) y de pollo (10\%), y en menor medida frutas (2\%), cereales (1\%) y legumbres (1\%). Este resultado no solo es entendible por las características del puerto, el cual puede hacer uso de los productos marítimos con suma facilidad, sino también porque el periodo vacacional coincide, como su nombre lo indica, con la Semana Santa, donde la mayoría de los creyentes de la religión cristiana evitan consumir carne. 
Perfil del turista nacional que consume alimentos durante Semana Santa en Mazatlán, Sinaloa

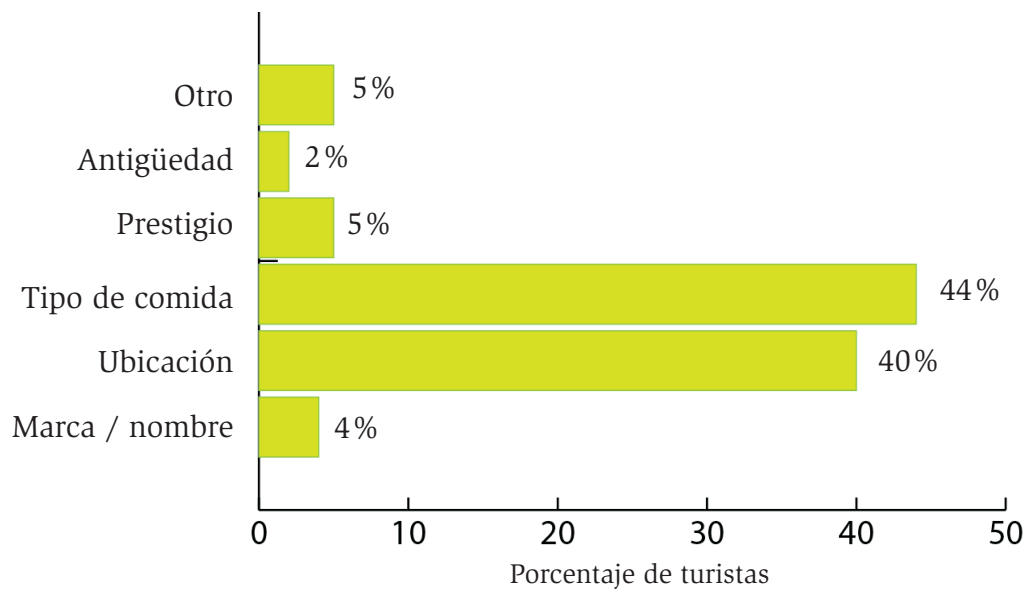

Gráfica 2. Motivos para elegir un restaurante

La elección de un restaurante para consumir alimentos en Mazatlán mostró que factores como el tipo de comida (44 \%) y su ubicación (40 \%) son los más importantes, muy por encima del prestigio (5\%), el nombre o marca (4\%), su antigüedad (2\%) u otros factores más (5\%).

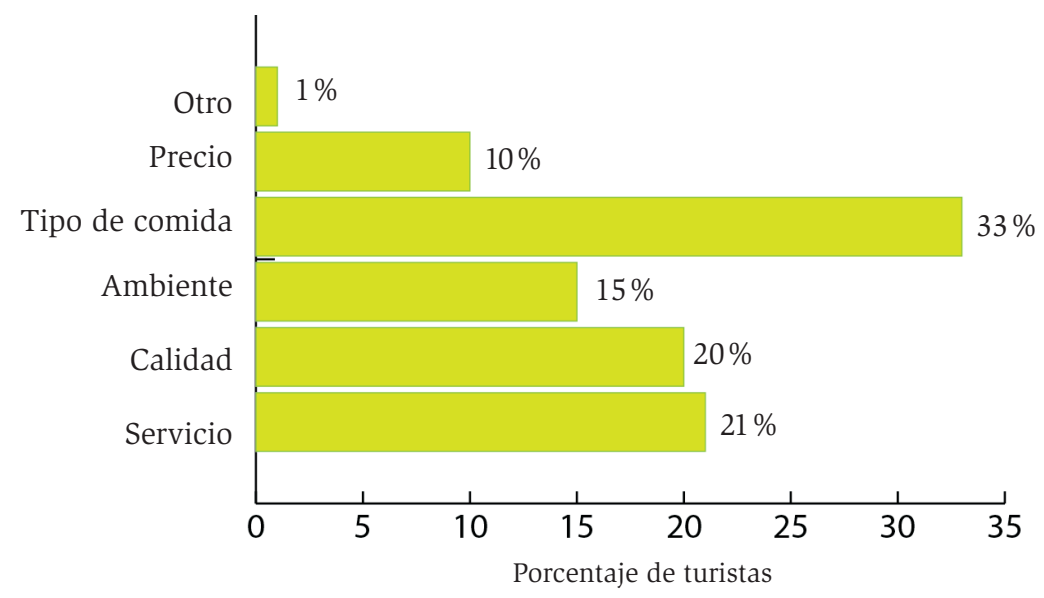


Por su parte, entre los motivos para regresar a un restaurante destaca nuevamente el tipo de comida (33\%), el servicio (21 \%), la calidad (20\%) y en menor medida el ambiente (15\%), el precio (10\%) y otros factores (1\%). Por lo tanto, si comparamos este resultado con el anterior tenemos que cuando eligen un restaurante, este tiene que especializarse, o por lo menos ofertar dentro de sus platillos aquellos alimentos elaborados a base de pescados y mariscos, ya que el tipo de comida es el factor fundamental para elegir y decidir regresar a un restaurante.

La gráfica 4 describe el gasto destinado al consumo de alimentos (gasto 1): $45 \%$ destina entre $\$ 101$ y $\$ 200 ; 25 \%$, entre $\$ 50$ y $\$ 100$ en promedio; mientras que $18 \%$ destina de $\$ 201$ a $\$ 300 ; 10 \%$, de $\$ 301$ a $\$ 500$ y solo dos personas consideran gastar más de $\$ 501$ para su alimentación.

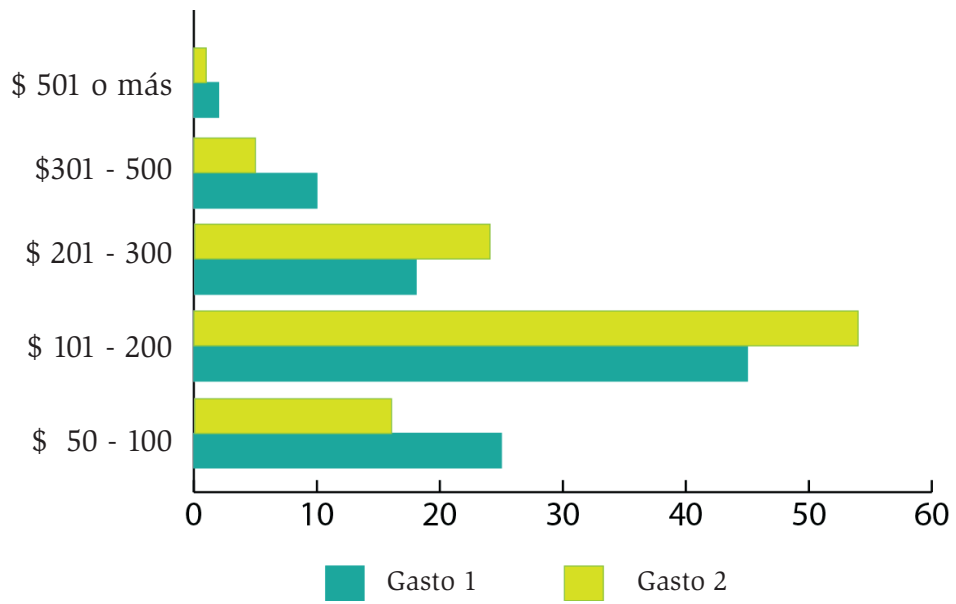

GRÁFICA 4. Gasto promedio por consumo de alimento

De manera similar, la mayoría de los turistas (54 \%) consideró que gastaría la misma cantidad ( $\$ 101$ a $\$ 200$ ) por un servicio completo de alimentos (gasto 2), es decir, tipo buffet. Por su parte, $24 \%$ estima como gasto por este servicio de $\$ 201$ a $\$ 300$, mientras que $16 \%$ gasta de $\$ 50$ a $\$ 100$, y en menor medida, de $\$ 301$ a $\$ 500$, únicamente $5 \%$ y solo una persona utilizaría más de $\$ 501$. Esto nos señala un ligero incremento en el gasto destinado si se ofrecen los alimentos como tipo buffet, en comparación con el que se eroga de forma individualizada. 


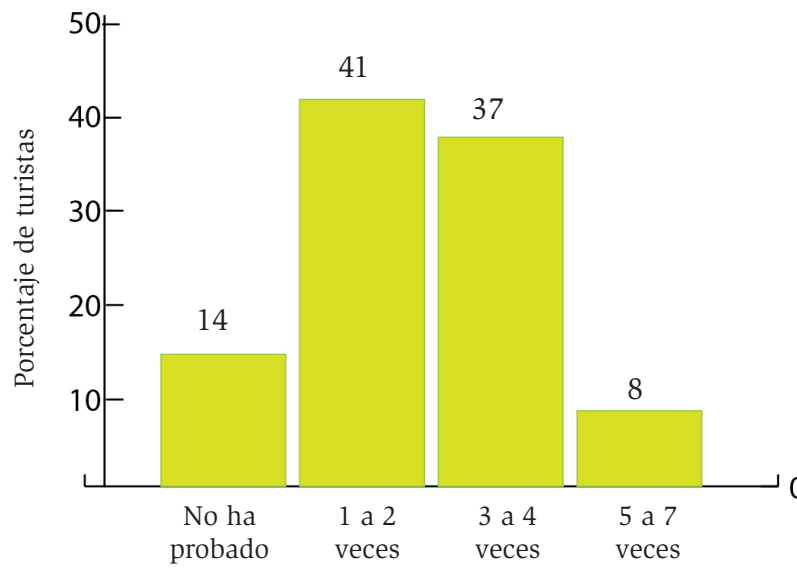

GráFICA 5. Frecuencia de consumo de platillos sinaloenses

La gráfica 5 muestra el consumo de platillos típicos sinaloenses: la mayoría dijo haberlos consumido (41\% de 1 a 2 veces, $37 \%$ de 3 a 4 veces y $8 \%$ de 5 a 7 ocasiones), y solo $14 \%$ no los había consumido.

Finalmente, en la cuarta parte de la entrevista a turistas nacionales que visitaron la ciudad y el puerto de Mazatlán durante la Semana Santa del 2012, por medio de una escala de Likert de 5 puntos, se midió si las expectativas de los visitantes se superaron en cuanto a la calidad de los alimentos, el servicio proporcionado y la limpieza de los mismos. Los resultados fueron los siguientes:

En lo que corresponde a la variable "calidad en los alimentos" (cA), la mayoría tiene una percepción satisfactoria, pues 41 y 36\% mostraron estar total o muy satisfechos respectivamente, y solo a 20 \% le pareció regular, mientras que el restante $3 \%$ dijo tener poco nivel de satisfacción. En cuanto al servicio otorgado por el personal que atiende en los diferentes establecimientos que ofertan alimentos (SP), la mayoría manifestó estar totalmente o muy satisfechos (25 y $42 \%$ ), superando su expectativa, mientras que $27 \%$ lo consideró regular y solo $6 \%$ expresó poca satisfacción (gráfica 6).

El renglón menos atractivo para los turistas fue el relacionado con la limpieza de los sitios donde consumían sus alimentos (LL), ya que la mayoría (51 \%) consideró que esta fue regular, $10 \%$ y $2 \%$ poco a nada satisfactoria, mientras que 27 y $10 \%$ consideraron que se cumplían sus expectativas mucho 


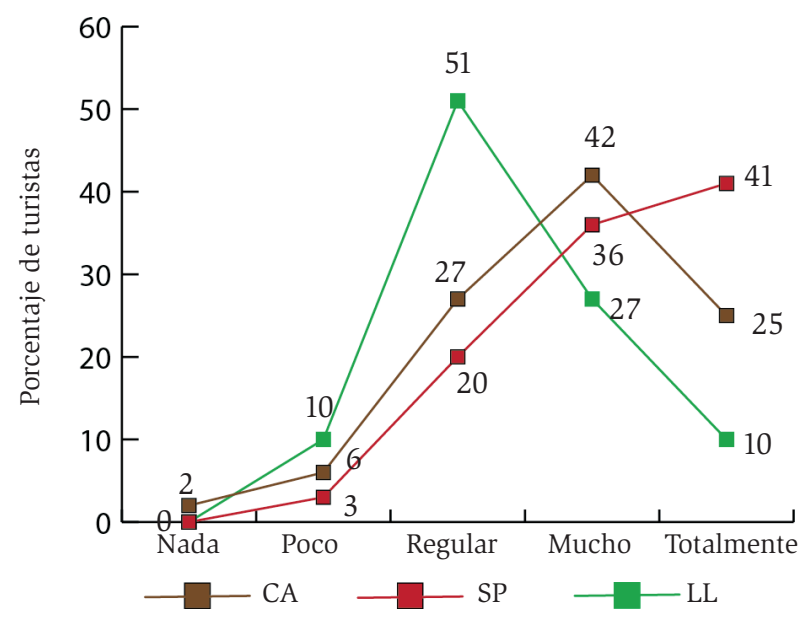

GRÁFICA 6. Expectativas sobre la calidad, servicio y limpieza de los alimentos

o totalmente. Esta situación permite observar que, si bien las expectativas por la limpieza están en un límite normal, es necesario que los negocios aumenten el nivel de satisfacción para contribuir de esa forma a una mejor imagen y percepción de la misma.

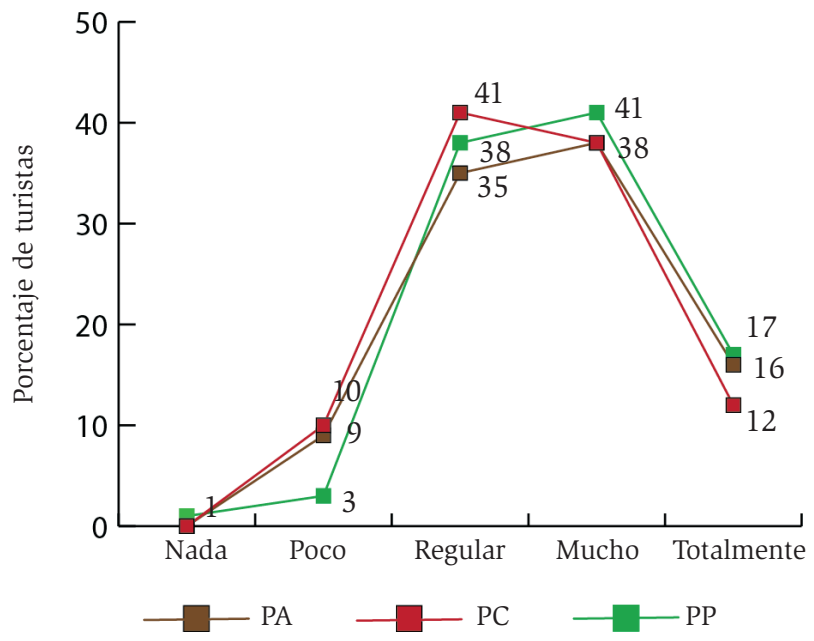




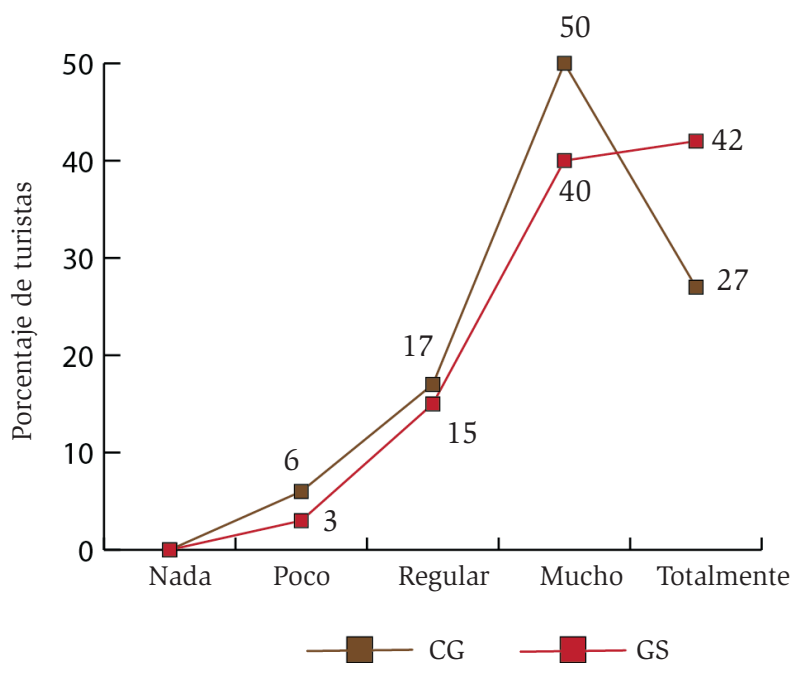

GRÁFICA 8. Expectativas sobre la gastronomía sinaloense

El área de análisis que se muestra en la gráfica 7 se asocia con el precio de los alimentos (PA); de nueva cuenta la mayoría mostró un alto nivel de satisfacción, pues 17 \% consideró su expectativa totalmente superada, mientras que 41 $\%$ en gran medida, es decir, muy por arriba de lo concebido antes de su arribo al puerto. Por su parte, $38 \%$ de los encuestados lo cree un precio regular, $3 \%$ estuvo poco satisfecho y solo 1 \% plasmó estar nada satisfecho con la situación.

En cuanto a las porciones de comida (PC), 41 \% las consideró regular, 38 \% contestó haber superado mucho sus expectativas, y $12 \%$ lo hizo de forma total. Solo 9 \% mencionó que su expectativa fue poco superada.

En este mismo orden de ideas, la presentación de los platillos (PP) también fue importante para los consumidores turistas, por lo que $38 \%$ se mostró muy satisfecho, $12 \%$ vio totalmente superadas sus expectativas, $41 \%$ lo concibió como algo regular y finalmente 9 \% señaló estar poco satisfecho con la forma en que presentaban los platillos. En resumen, tanto el precio como las porciones y la presentación de los platillos recibieron altas calificaciones en sus expectativas por parte de los entrevistados, a pesar de que en el periodo de Semana Santa los servicios de alimentos son muy demandados en Mazatlán.

Sobre la gastronomía sinaloense (gráfica 8), desde el punto de vista de su variedad (VG), la mayoría (50 \%) expresó que sus expectativas había sido superadas 
en gran medida, 27 \% mostró una satisfacción total. A su vez, 17 \% certificó la variedad como regular, y solo 6 \% manifestó poca satisfacción.

El último atributo medido fue la expectativa que tenían los turistas en cuanto al consumo de alimentos sinaloenses (Gs); la mayoría de ellos mencionó que estas fueron superadas (40\%), mientras que de forma total $42 \%$, solo $15 \%$ la juzgó regular y, finalmente, 3 \% estimó que sus expectaciones no fueron superadas. Este resultado representa un buen sabor de boca no solo para los turistas que tuvieron la oportunidad de consumir platillos típicos de la región, sino también para el destino turístico mazatleco, que demuestra un enorme potencial gastronómico para su consumo y promoción como parte de los atractivos turísticos locales.

\section{Conclusión}

El presente estudio muestra una perspectiva de lo importante que es para un destino turístico, en este caso Mazatlán, su oferta gastronómica, ya que se encontró que gran parte de los visitantes acude a un restaurante a consumir sus alimentos, con una marcada preferencia por aquellos elaborados a base de pescados y mariscos. Además, su característica económica y geográfica le otorga una gran ventaja, pues al ser también un importante puerto pesquero tiene la oportunidad de ofrecer este tipo de alimentos frescos y con una amplia variedad de presentaciones.

Es importante mencionar el gasto por consumo de alimentos que realizan los turistas, (entre $\$ 100$ y $\$ 300$ en promedio por persona para satisfacer esta necesidad), gasto que, multiplicado por cada consumo de alimentos al día y por cada turista que arriba al puerto durante Semana Santa ofrece un panorama más claro de la magnitud de la derrama económica en el destino y en los establecimientos que ofrecen el servicio de alimentos.

Como parte del perfil del turista nacional que visita Mazatlán durante Semana Santa, destaca el predominio de visitantes provenientes de la región, es decir, de municipios sinaloenses y de estados cercanos a Sinaloa, asimismo, la mayoría de ellos ya había visitado el destino, por lo que forma parte de un segmento que muestra cierto nivel de lealtad. Este perfil se complementa al considerar a este tipo de turistas como personas con un gusto predilecto por alimentos provenientes del mar (pescados y mariscos), que gustan de consumir 
en restaurantes, con un gasto promedio que va de los $\$ 100$ a $\$ 300$ por persona, $\mathrm{y}$, por último, que poseen conocimiento sobre algunos platillos sinaloenses, por lo que los consumen de forma constante durante su estancia.

Cabe mencionar que el único punto por reforzar tiene que ver con la imagen y limpieza de los establecimientos que ofrecen servicio de alimentos en Mazatlán, pues fue el rubro que menos superó las expectativas de los visitantes. Por tal motivo, se recomienda tanto a la Cámara Nacional de la Industria de Restaurantes y Alimentos Condimentados local como a la Secretaría de Turismo de Sinaloa y al gobierno municipal, elaborar un programa conjunto que detecte, capacite y supervise la limpieza en aquellos sitios donde esta sea insuficiente.

Por otro lado, si bien Mazatlán no es considerado un destino donde se pueda desarrollar el turismo gastronómico, su comida tiene los atributos necesarios para convertirse en una opción más de su oferta turística, pues cuenta con producción de la materia prima indispensable para la elaboración de los alimentos que requiere la industria turística (en este caso del turismo de sol y playa), ya que "la producción debe estar en condiciones de aprovisionar a los restaurantes para evitar traer productos del exterior y así dejar los beneficios en el lugar. De esa forma se evita el egreso de recursos hacia otros lugares de producción y también se evita la polución creada por los sistemas de transporte para el abasto" (Schlüter, 2006: 124).

Mazatlán es un destino de sol y playa que debe ser promocionado junto con su gastronomía, vista como parte de la oferta turística hacia sus visitantes, ya que tomar la gastronomía típica como atractivo turístico representa un importante elemento diversificador que le otorga al visitante la oportunidad de conocer y disfrutar de las tradiciones culinarias del puerto, entre las que sobresalen los alimentos elaborados a base de pescados y mariscos, por lo que se debe levantar un censo con fines mercadológicos y turísticos para conocer qué platillos son más preferidos por este tipo de personas. Aunado a ello, se recomienda la profundización de estudios como este para conocer mejor el comportamiento de los turistas en cuanto al consumo de alimentos en otras épocas del año, el gasto que le destinan y el impacto que puede tener la gastronomía típica sinaloense en su mente como parte del cuidado y promoción del patrimonio de la localidad. 


\section{Fuentes consultadas}

Anaya Ortiz, Julia Sderis y Alejandro Palafox Muñoz (2010). "El perfil del turista internacional de Cozumel a partir de la construcción de su capital simbólico”. Teoría y Praxis, 8, 171-185.

Blanco, Javier (2010). "El valor del turismo gastronómico en el contexto mundial”. I Congreso Europeo del Turismo y la Gastronomía. Madrid: Organización Mundial del Turismo.

Clemente Ricolfe, José Serafín, et al. (2008). "Actitud hacia la gastronomía local de los turistas: dimensiones y segmentación de mercado”. Pasos. Revista de Turismo y Patrimonio Cultural, 6 (2), 189-198.

Cabarcos Novás, Noelia (2006). Promoción y venta de servicios turísticos. Comercialización de Servicios Turísticos. Madrid: Ideaspropias Editorial, $304 \mathrm{p}$.

Cestur (2011). Análisis de la lealtad del consumidor de destinos turísticos en México [en línea]. México: Centro de Estudios Superiores en Turismo, 29 pp. Disponible en: http://cestur.sectur.gob.mx/PDFs/AnalisissobrelalealtaddelconsumidosdedestinosturisticosenMexico(2011).pdf

Flavián Blanco, Carlos y Carmina Fandos Herrera (2011). Turismo gastronómico. Estrategias de marketing y experiencias de éxito. Zaragoza: Prensas Universitarias de Zaragoza/Universidad de Zaragoza, 266 p.

Gamboa Mora, Patricia (2007). "Una experiencia de turismo cultural: los turistas nacionales y extranjeros en el centro histórico de Mazatlán durante Semana Santa 2006”. Revista Claves del Turismo, 1, 40-62.

González Turmo, Isabel (1999). "La dimensión social de la alimentación: consideraciones metodológicas”, en Alimentación y cultura. Actas del Congreso Internacional, 1998. Madrid: Museo Nacional de Antropología, 245-257.

Guerrero Ocampo, Miguel, Agustín Santiago Moreno y Carlos Bouza Herrera (2009). "El mercado turístico de Acapulco: estudio del perfil de los turistas”. Redmarka-Ciecid, Revista Académica de Marketing Aplicado [en línea], 2 (1), 77-110. Disponible en: http://www.cienciared.com. ar/ra/usr/39/702/redmarka_n2_v1pp77_110.pdf [2012, 3 de octubre]. Gurría Di-Bella, Manuel (1994). Introducción al Turismo. México: Trillas, 136 p. 
Holloway, J.C. (1997). El negocio del turismo. Trad. Héctor de Lille. México: Editorial Diana, $447 \mathrm{p}$.

INEGi (2010). Anuario Estadístico de Sinaloa. México: Instituto Nacional de Estadística y Geografía.

Lizárraga Morales, Omar (2005). "La era de la abundancia estadounidense y el desarrollo del turismo anglosajón en Mazatlán”. Arenas, 8, 51-75.

(2007). "La religión transnacional como vehículo turístico". Claves del Turismo, 2, 51-61.

Martínez Jasso, Irma y Pedro A. Villezca Becerra (2005). "La alimentación en México. Un estudio a partir de la encuesta nacional de ingresos y gastos de los hogares y de las hojas de balance alimenticio de la FAo". Ciencia UANL, VIII, abril-junio, 196-208.

Nunes dos Santos, Cristiane (2007). "Somos lo que comemos. Identidad cultural, hábitos alimenticios y turismo”. Estudios y Perspectivas en Turismo, 16, 234-242.

Quesada Castro, Renato (2007). Elementos del turismo. San José: Editorial Universidad Estatal a Distancia.

Rodríguez, Emira, Karen Requena y José Muñoz (2009). “La percepción turística del mercado español hacia Latinoamérica”. Revista de Artes y Humanidades UNICA, 10 (2), 133-166.

Santamaría Gómez, Arturo (2002). El nacimiento del turismo en Mazatlán 19231971. Culiacán: Universidad Autónoma de Sinaloa, 137 p.

------ (2005). Del alba al anochecer. El turismo en Mazatlán (1972-2004). Culiacán: Universidad Autónoma de Sinaloa, 298 p.

Santamaría Gómez, Arturo y Adriana Barbosa Jasso (2006). Estudio Comparativo de Playas: Mazatlán, Acapulco, Cancún y Los Cabos (1970-2005). Culiacán: Universidad Autónoma de Sinaloa, 152 p.

Santamaría Gómez, Arturo y Silvestre Flores Gamboa (2012). Escenarios de violencia e inseguridad en los destinos turísticos. Mazatlán como estudio de caso. Mazatlán: Cuerpo Académico Desarrollo Regional y Movimientos Migratorios-Universidad Autónoma de Sinaloa, 170 p.

Santamaría Gómez, Arturo y Yasser Orlando Espinoza García (2011). Norteamericanos en el paraíso. Inversiones y turistas en Mazatlán (Siglo XX). Mazatlán: Cuerpo Académico Desarrollo Regional y Movimientos Migratorios-Universidad Autónoma de Sinaloa, 127 p. 
Santoyo Parroquín, Yuriria Jazmín (1999). "Análisis etnográfico de la semana santa en Mazatlán, Sinaloa”. Turismo y Cultura, 217-224.

Sectur (2011). Encuesta del Gasto en Turismo en Hogares 2011 [en línea]. México: Secretaría de Turismo, 48 p. Disponible en: http://datatur.sectur. gob.mx/work/sites/datatur/resources/LocalContent/273/24/EncuestGastTurHog2011.pdf [2012, 3 de septiembre].

Schlüter, Regina G. (2006). Turismo y patrimonio gastronómico. Una perspectiva. Buenos Aires: Centro de Investigaciones y Estudios Turísticos. 